\title{
Raven Calls Indicate Sender's Neural State
}

\author{
Zixuan HUANG ${ }^{1,2 *}$, Zhilong WANG ${ }^{3 *}$, Jun $\mathrm{XIE}^{4 *}$, Greg MIRT ${ }^{5 *}$, Chengying YAN ${ }^{4}$,
} Jing $\mathrm{ZHONG}^{4}$, Xianli DENG ${ }^{4}$, Fangfang $\mathrm{LIU}^{6}$, Chunlin $\mathrm{ZHOU}{ }^{1,2^{\wedge}}$, Fan $\mathrm{XU}^{7 \wedge}$

1. School of Geography Science, Nanjing Normal University, Jiangsu, CHINA 210023

2. Jiangsu Center of Collaborative Innovation in Geographical Information Resource Development and Application, Jiangsu, CHINA 210023

3. The Engineering \& Technical College of Chengdu University of Technology, Sichuan, CHINA 614000

4. Department of Pharmacy, Chengdu Medical College, Sichuan, 610500

5. Neuro Occupational Activity Centre Novo mesto, Slovenia, EU

6. Southwest Minzu University, Sichuan, CHINA, 610041

7. Nanjing Institute of Tourism and Hospitality, Jiangsu, CHINA, 211100

8. Department of Public Health, Chengdu Medical College, Sichuan, CHINA 610500

*These authors contributed equally

Co-corresponding Authors

Prof Chunlin ZHOU

Prof Fan XU

School of Geography Science

Department of Public Health

Nanjing Normal University

Chengdu Medical College

Nanjing

Jiangsu Province

Chengdu

CHINA

Sichuan Province

210023

CHINA

Tel:+862585891742

610500

Email: clzhou@njnu.edu.cn

Tel: +862862739397

Email:xufan@cmc.edu.cn 


\begin{abstract}
Vocal communication accounts for dominantly percentage within animal species. The information of vocal samples contains not only the amplitude of objects, but also the emotional states behind it. However, to extract the emotion state behind the sound remains controversial. Here we introduce an artificial network method, the Back Propagation Neural Network, BPNN, to classify the emotional states behind the sound. The results disclosed the behaviour categories, including alarm, flight, begging and singing which has been successfully classified. This artificial intelligence classification may aid us to distinguish the ecological categories via animal vocal communication and to discover its significance of evolution and nature.
\end{abstract}

Key words: vocal communication, raven, artificial intelligence, Back Propagation Neural Network, emotional states.

\title{
Background
}

The sound is one of universal phenomenon, which does not only exist in numerous nature phenomenon [1-3], but also appear in animal communication in species or across species $[4,5]$. From the perspective of physics, the sound is generated by the amplitude of object, which determines the frequency and intensity [6-8]. According to evolution, the ability of adaption and survival skills of species had been constructed and strengthened for surviving, for example: track the life source, avoid the harmful events and defend the territory $[9,10]$.

With respecting the animal communication, there are three domain categories, sound, behaviour and the order [11-14]. The pronunciation process of animal depends on a series of muscles interaction, including breathing muscles, mouth muscles and throat muscles and the amplitude of vocal cord $[15,16]$. Vocal communication accounts for dominant percentage in species $[17,18]$. Sound response on the stimulation is the best indicator of sender's emotional state under the specific circumstances, such as nervousness, sadness, happiness, repulsiveness, angriness and afraid $[19,20]$.

Interestingly, the raven, an intelligent avian, has been used in many specific mind and brain studies for decades [21-23]. For example, the raven's memory predict future [24], sequence tool used to get the award [25], the awareness of density of article[26], and so on. Ravens' call often appears frequently during their foraging[27], defend the territory[28], rutting and mating[29], infant parenting[30]. Even though we can hear various different callings, however, to classify the emotional states behind the sound remains unclear. Here are four behaviour validated related calls of raven are hypothesized to be directly different among acoustic parameters, providing cues to 
emotional category recognition. Therefore, we use the method, termed as "Back Propagation Neural Network, BPNN", to disclose the emotion behind the sound. This may be helpful to us to understand each other better, not only in same species, but also across species.

\section{Method}

\section{Data Source}

Total 851 sound samples of raven have been downloaded from the website (https://www.xeno-canto.org/species/Corvus-corax ) and their detail information sorted into excel file, totally 11 variables included: "Common name, scientific name, recording duration, recording person, date and time of recording, country, location, altitude, tweet type, behaviour marker, audio file classification number". The variable "type" described the behaviour profiles according to the current situation, including alarm, fighting, flying, begging, and singing. Furthermore, the "Remarks" listed the detail raven's behaviours. Aim to reduce the noise impact and enhance the discrimination effect, the sound samples, which contain more than 2 raven's sounds, have been excluded. Four different categories of sound samples (alarm, singing, fighting and begging) have been selected. Consequently, there were only 100 sound samples recruited in the final data analysis.

\section{Acoustic Parameters Extraction}

Here we use the MATLAB script to extract the acoustic parameters [31, 32]. Including: (1) Mean fo(Hz), (2) Maximum fo(Hz), (3) Minimum fo(Hz), (4) Range fo(Hz), (5) Start fo(Hz), (6) End fo(Hz), (7) fo at the half of call duration (mid fo Hz), (8) slope from fo start of the call to the fo maximum (slope S-M; Hz/s), (9) Slope from maximum fo to the end of the call (Slope M-E; Hz/s), (10) Inflection rate (number of frequency changes/s), (11) Harmonicity (HNR, dB), (12) Jitter (the absolute ; fo difference between consecutive fo measurements/ the average period), (13) fo variation (sum of all fo changes measured/ call duration, Hz), (14) amplitude range (maximum $\mathrm{dB}-$ minimum $\mathrm{dB}$ ).

\section{Data Analysis}

According to the raven's call recorded under the different circumstances, the biological meanings may be presented differently. Therefore, we selected Back Propagation Neural Network (BPNN) method [33-35]. A typical BPNN consists of the input layer, hidden layer and output layer (Figure 1). 


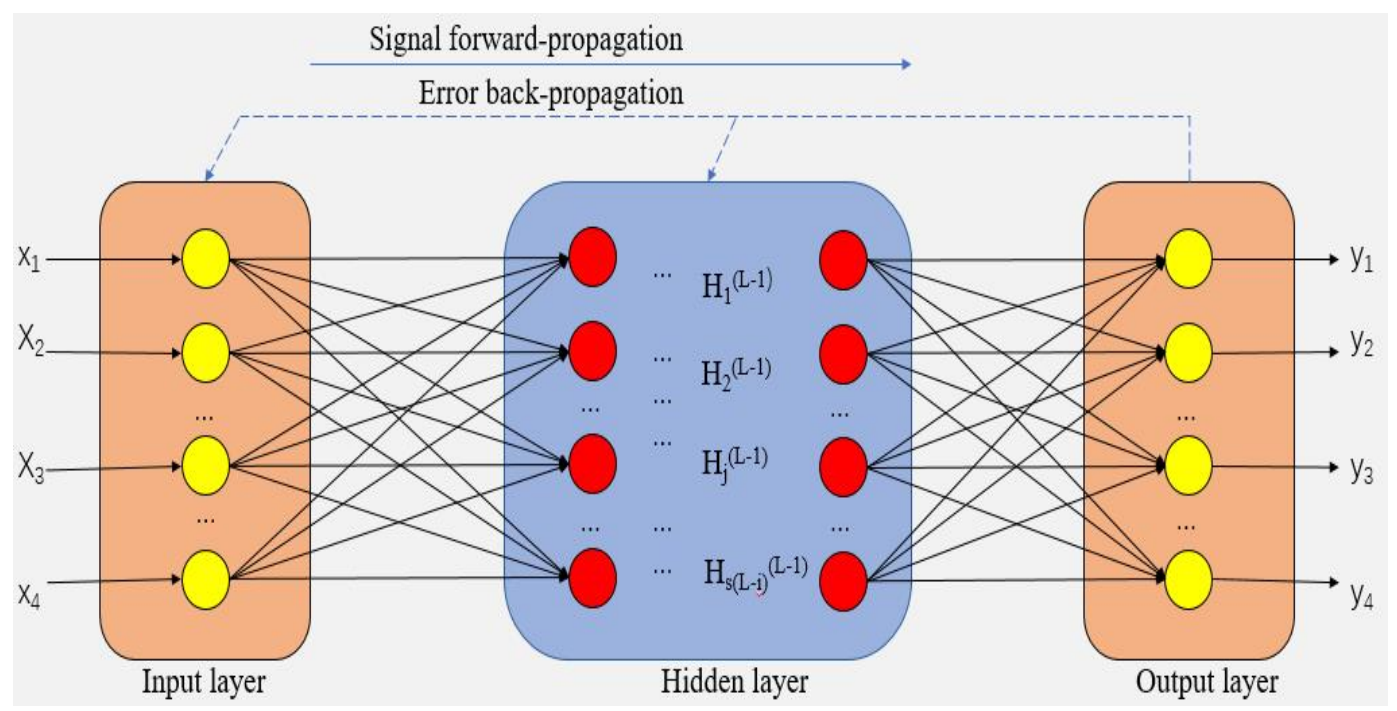

Figure 1. BP Neural Network structure

\section{Result}

Totally we have selected four different categories call of raven, including alarm, begging, fighting and singing. The descriptive acoustic parameters extracted from MATLAB, please see Table 1.

\section{Table 1 Four different Call Sound Parameters}

\begin{tabular}{|c|c|c|c|c|c|c|c|c|c|c|c|c|}
\hline \multirow{2}{*}{ Parameters } & \multicolumn{3}{|c|}{ ALARM } & \multicolumn{3}{|c|}{ FLIGHT } & \multicolumn{3}{|c|}{ BEGGING } & \multicolumn{3}{|c|}{ SONG } \\
\hline & $\mathrm{N}$ & means & s.dev. & $\mathrm{N}$ & means & s.dev. & $\mathrm{N}$ & means & s.dev. & $\mathrm{N}$ & means & s.dev. \\
\hline $\begin{array}{l}\text { Amplitude } \\
\text { range }\end{array}$ & 10 & 0.7107576 & 0.5005164 & 74 & 1.0111211 & 0.572916 & 6 & 0.9334848 & 0.746398 & 10 & 0.894717 & 0.624794 \\
\hline fo variation & 10 & -2.676767 & 6.7065086 & 74 & -139.71842 & 1094.613 & 6 & -8.852867 & 18.1973 & 10 & -68.3103 & 144.6527 \\
\hline Start $f_{o}$ & 10 & $-1.83 \mathrm{E}-05$ & $3.86 \mathrm{E}-05$ & 74 & 0.0002924 & 0.002695 & 6 & $-2.035 \mathrm{E}-05$ & 4.98E-05 & 10 & $-8.2 \mathrm{E}-05$ & 0.000261 \\
\hline Harmonicity & 10 & -21.22948 & 7.2070505 & 74 & -21.673169 & 5.745358 & 6 & -25.54435 & 8.567675 & 10 & -19.8766 & 6.758902 \\
\hline Inflection rate & 10 & $2.46 \mathrm{E}-09$ & $5.63 \mathrm{E}-09$ & 74 & $-4.60 \mathrm{E}-10$ & $1.78 \mathrm{E}-08$ & 6 & $-1.37 \mathrm{E}-09$ & $3.76 \mathrm{E}-09$ & 10 & $1.50 \mathrm{E}-08$ & $4.78 \mathrm{E}-08$ \\
\hline Jitter & 10 & 2.81E-09 & $6.22 \mathrm{E}-09$ & 74 & $-1.40 \mathrm{E}-09$ & $1.06 \mathrm{E}-08$ & 6 & $-1.16 \mathrm{E}-09$ & 4.10E-09 & 10 & $1.07 \mathrm{E}-08$ & $3.35 \mathrm{E}-08$ \\
\hline End $f o$ & 10 & 0.0044069 & 0.0105198 & 74 & -0.001516 & 0.010735 & 6 & -0.001943 & 0.0112 & 10 & 0.001276 & 0.003853 \\
\hline Maximum fo & 10 & 0.3562669 & 0.2590624 & 74 & 0.4943541 & 0.284507 & 6 & 0.4442582 & 0.350765 & 10 & 0.437178 & 0.31567 \\
\hline Mean $f o$ & 10 & $-6.41 \mathrm{E}-06$ & $2.284 \mathrm{E}-05$ & 74 & -0.0007544 & 0.006447 & 6 & $-1.181 \mathrm{E}-05$ & $1.73 \mathrm{E}-05$ & 10 & -0.00165 & 0.003674 \\
\hline Middle $f o$ & 10 & -0.000305 & 0.0200354 & 74 & -0.0023711 & 0.037413 & 6 & 0.0065875 & 0.017132 & 10 & -0.00995 & 0.050983 \\
\hline Minimum $f_{o}$ & 10 & -0.354492 & 0.2501229 & 74 & -0.5167672 & 0.293957 & 6 & -0.4892283 & 0.405914 & 10 & -0.45754 & 0.312752 \\
\hline Range of $f o$ & 10 & 0.7107576 & 0.5005164 & 74 & 1.0111211 & 0.572916 & 6 & 0.9334848 & 0.746398 & 10 & 0.894717 & 0.624794 \\
\hline Slope $M-E^{\#}$ & 10 & $-2.27 \mathrm{E}-06$ & $5.011 \mathrm{E}-06$ & 74 & $-1.191 \mathrm{E}-06$ & $1.87 \mathrm{E}-06$ & 6 & $-7.04 \mathrm{E}-07$ & $1.43 \mathrm{E}-06$ & 10 & $-3.9 \mathrm{E}-06$ & 4.69E-06 \\
\hline Slope S-M & 10 & $3.72 \mathrm{E}-07$ & $2.99 \mathrm{E}-07$ & 74 & $3.493 \mathrm{E}-06$ & $6.68 \mathrm{E}-06$ & 6 & $1.131 \mathrm{E}-06$ & $1.4 \mathrm{E}-06$ & 10 & $3.23 \mathrm{E}-06$ & 4.69E-06 \\
\hline
\end{tabular}

\# One-way analysis, $\mathrm{F}=3.39$, $\mathrm{df}=3, \mathrm{P}=0.0211$;

By meaning of BPNN method, the biological meanings of raven's call have been classified. Firstly, we have selected the training samples: 14 sound parameters were used. We tested first 100 sound samples. Namely, all data imported training dataset. Secondly, pre-processing: Due to realistic complexity, we used the standard process of deviation normalization $(\mathrm{x} . \mathrm{std} .=(\mathrm{x}-\mathrm{min}) /(\max -\mathrm{min}))$ to perform the better neural 
network and to reduce the negative effect of over fit. The new data was constructed via dimensionless processing. Thirdly, primary parameter selection: The number of input layer units was 14 . There was only one output layer neuron. Then, the number of hidden layer neurons was 12. Details please see Figure 2.

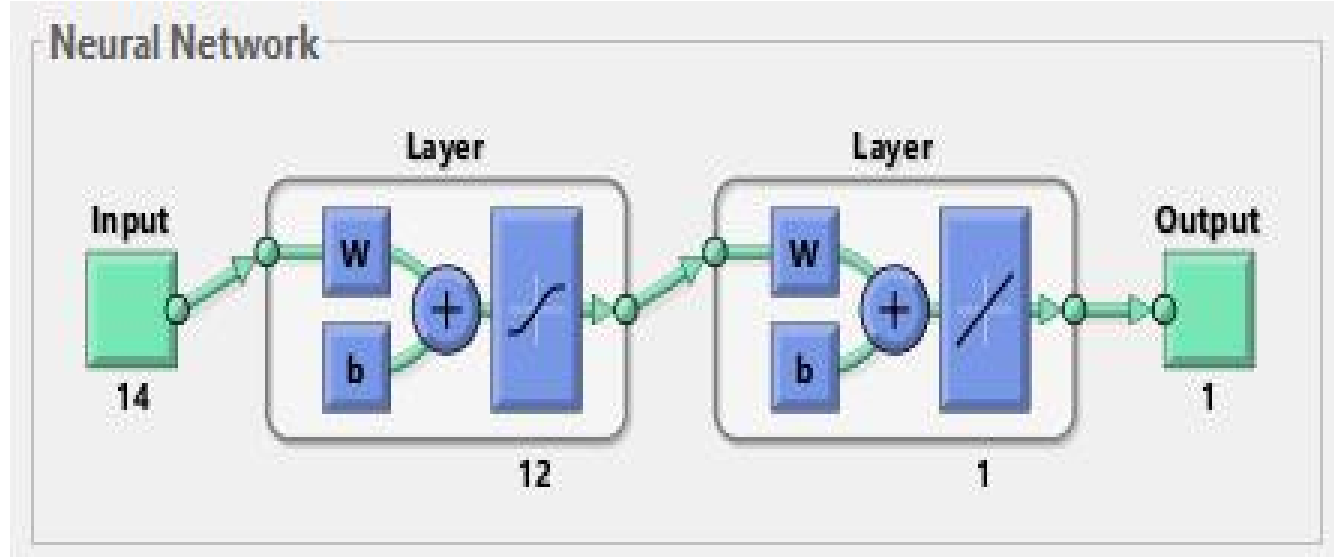

Figure 2. Neural Network Pattern

The unique purpose of BPNN construction is to classify. Double transfer sigmoid function and linear were used. The results were presented well when used "tansig" function in the input layer-hidden layer and linear function in the hidden layer-output layer. Furthermore, the "trainlm" method (combined gradient descent method with Newton method) was used for training, which terminated when achieved the best effect. The details of training performance process please see Figure 3.

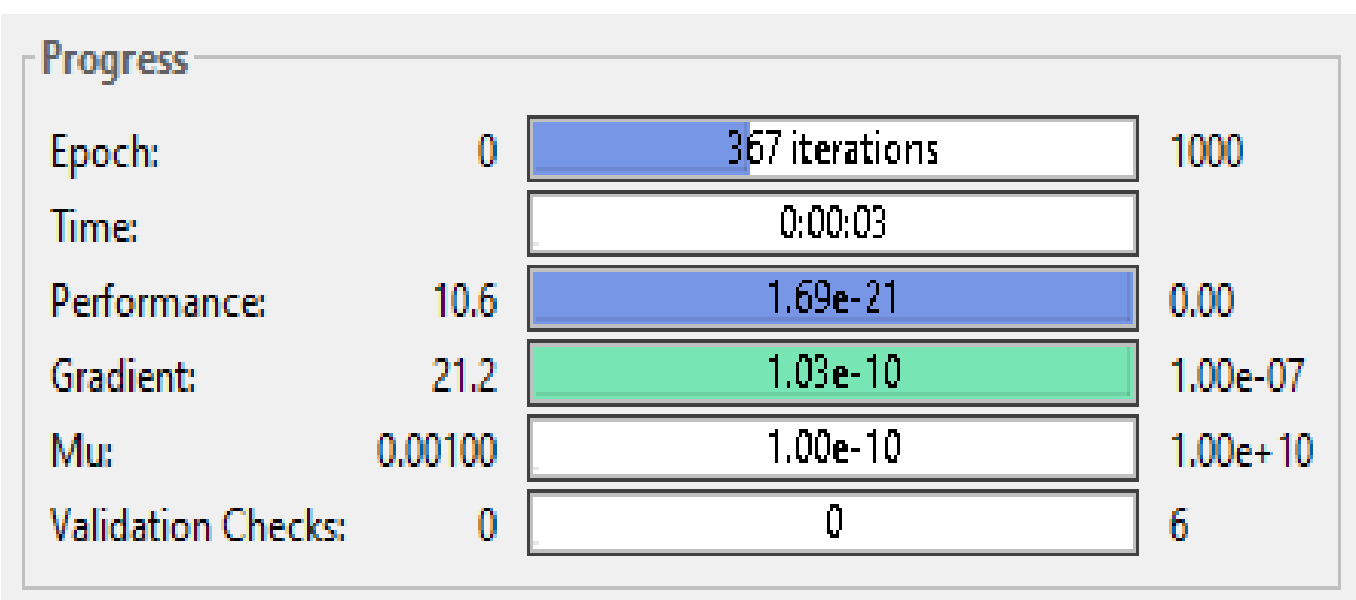

Figure 3. Training Process

Fourth, Validation the prediction effect: the training network was used to predict the types of raven calls, to detect the prediction and promotion ability of the network, and to get the classification of network simulation and actual classification. The detail comparison results please see figure 4 . 


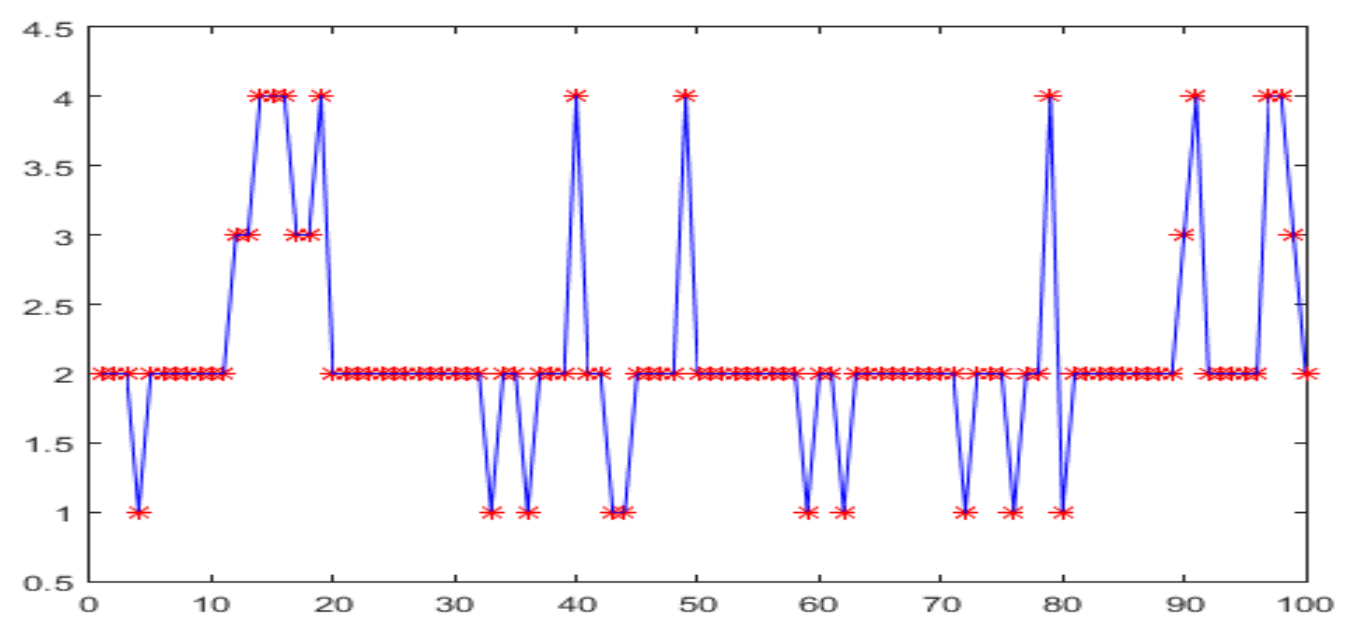

Figure 4. Prediction effect

Figure legend: red star stands for actual classfication, blue line stands for stimulation classfication

The overall trend of the predicted value was close to the actual value. It can be inferred that the prediction of the model is more accurate, while it has a certain reference value. The results disclosed that the average absolute error and the average relative error of the actual classification and the predicted classification were 1.0e-09 and $1.0 \mathrm{e}-10$, respectively.

\section{Discussion}

The sound is the best indicator to reflect the property of the object [36, 37]. From the physical perspective, the size of sound depends on the frequency of object, and the pitch of sound depends on the amplitude of object [38, 39].

With respect to the animal communication, the pronunciation is not only depending on a series of pronounced organ coordination work [40-42], but also depending on the control of central nervous system [43]. The animals have been adapted to the environment after a long time evolution process via a series of successful constructed conditional reflexes [44]. It is obvious that the emotional information maybe hide behind the vocal communication.

The raven is recognized as the most intelligent avian [45, 46]. It was being used in mind, sequenced tool used test, awareness, learning and memory researches. More interestingly, Markus et al distinguished the gender and age of raven [47], and reported that ravens can predict future via their memory [24].

There are four behaviour categories of raven's call, including alarm, begging, flighting and singing. It is necessary to distinguish the specific behaviouristic category. Thanks to BPNN method, their behaviour categories have been classified successfully.

The abundance information is contained within the sound in animal communication. There is not only the amplitude information of object, but also 
embedded emotional state. For example, professional police can distinguish the criminal suspects via sophisticated conservation due to their unstable irregular speaking [48]; experienced hunter can determine the degree of hunger of animal [49].

To distinguish the emotional state behind the sound is of great significance. Through the emotion information behind the ravens' sound, we can get better understanding of their biological meanings. For instance, the alarm call often occurs on the occasion of dangerous events approaching, it is good for avoiding the harmful events and surviving $[50,51]$. The flight call occurs on the situation of fighting process; the failure subject presents submission to dominant subject to avoid the further damage on its body or relationship [52, 53]. Moreover, the begging call happens when sub-dominant subject paying / begging for the life sources, such as the food, resting place etc $[54,55]$. As for the singing; this may arise on the situation of pleasant animal communication, as for the specific motivation remains controversial.

Taken together, this artificial network could be helpful of trying to distinguish the behaviour categories of different raven's sounds. It can strengthen our understanding on their biological and evolution meanings.

\section{Conclusion}

Taken together, we herewith disclose the method of BPNN could be the promising candidate to classify the category of raven's call. Furthermore, this may help us to investigate more in-depth of neurobiology of raven's mind.

\section{Abbreviations List:}

BPNN: Back Propagation Neural Network

\section{Declarations}

\section{Ethic Statement}

Not applicable.

\section{Consent for publication}

Not applicable

\section{Availability of Data and material}

All data will be open for reasonable request.

\section{Funding}

This work was supported by Chengdu Medical College Natural Science Foundation (CYZ18-08, CYZ18-20, CYZ18-33)

\section{Competing interests}

The authors declare that they have no competing interests.

Authors' Contribution: X.F drafted the general idea and drafted the manuscript with 
HZX; XJ, ZJ, YCY and DXL collected the sound data from xeno-canto website and data clean.HZX and WZL performed the MATLAB analysis.

Acknowledgement: We thanks Dr Huang Kun provided the valuable data mining strategy and Peter proofreading and ensures the general language quality.

\section{Reference}

1. Geipel, I., et al., Noise as an informational cue for decision-making: the sound of rain delays bat emergence. J Exp Biol, 2019.

2. Weinzierl, S., et al., Sound power and timbre as cues for the dynamic strength of orchestral instruments. J Acoust Soc Am, 2018. 144(3): p. 1347.

3. Caldwell, M.T., P. Jiradejvong, and C.J. Limb, Effects of Phantom Electrode Stimulation on Vocal Production in Cochlear Implant Users. Ear Hear, 2018.

4. Fedurek, P. and K.E. Slocombe, Primate vocal communication: a useful tool for understanding human speech and language evolution? Hum Biol, 2011. 83(2): p. 153-73.

5. Kraus, N. and J. Slater, Beyond Words: How Humans Communicate Through Sound. Annu Rev Psychol, 2016. 67: p. 83-103.

6. Popper, A.N., ASA S3/SC1.4 TR-2014 Sound Exposure Guidelines for Fishes and Sea Turtles: A Technical Report prepared by ANSI-Accredited Standards Committee S3/SC1 and registered with ANSI. 2014: Springer, Cham.

7. Jose, K., A. Chatterjee, and A. Gupta, Acoustics of Idakka: An Indian snare drum with definite pitch. J Acoust Soc Am, 2018. 143(5): p. 3184.

8. Ladich, F. and H. Winkler, Acoustic communication in terrestrial and aquatic vertebrates. J Exp Biol, 2017. 220(Pt 13): p. 2306-2317.

9. Sistrunk, J.R., et al., Survival of the Fittest: How Bacterial Pathogens Utilize Bile To Enhance Infection. Clin Microbiol Rev, 2016. 29(4): p. 819-36.

10. Chen, L., et al., Acute exposure to PBDEs at an environmentally realistic concentration causes abrupt changes in the gut microbiota and host health of zebrafish. Environ Pollut, 2018. 240: p. 17-26.

11. Zhou, W., et al., Seasonal and reproductive variation in chemical constituents of scent signals in wild giant pandas. Sci China Life Sci, 2019.

12. Jiang, T., et al., Bats increase vocal amplitude and decrease vocal complexity to mitigate noise interference during social communication. Anim Cogn, 2019.

13. Dakin, R. and R. Montgomerie, Deceptive copulation calls attract female visitors to peacock leks. Am Nat, 2014. 183(4): p. 558-64.

14. Saul-Gershenz, L., et al., Deceptive signals and behaviors of a cleptoparasitic beetle show local adaptation to different host bee species. Proc Natl Acad Sci 
U S A, 2018. 115(39): p. 9756-9760.

15. Zhang, Z., Mechanics of human voice production and control. J Acoust Soc Am, 2016. 140(4): p. 2614.

16. Alipour, F., D.A. Berry, and I.R. Titze, A finite-element model of vocal-fold vibration. J Acoust Soc Am, 2000. 108(6): p. 3003-12.

17. Frohlich, M., Taking turns across channels: Conversation-analytic tools in animal communication. Neurosci Biobehav Rev, 2017. 80: p. 201-209.

18. Rojas, B., Behavioural, ecological, and evolutionary aspects of diversity in frog colour patterns. Biol Rev Camb Philos Soc, 2017. 92(2): p. 1059-1080.

19. Faure, P.A. and R.R. Hoy, The sounds of silence: cessation of singing and song pausing are ultrasound-induced acoustic startle behaviors in the katydid Neoconocephalus ensiger (Orthoptera; Tettigoniidae). J Comp Physiol A, 2000. 186(2): p. 129-42.

20. Sachs, M.E., et al., Decoding the neural signatures of emotions expressed through sound. Neuroimage, 2018. 174: p. 1-10.

21. Jonsson, K.A., P.H. Fabre, and M. Irestedt, Brains, tools, innovation and biogeography in crows and ravens. BMC Evol Biol, 2012. 12: p. 72.

22. Cnotka, J., et al., Extraordinary large brains in tool-using New Caledonian crows (Corvus moneduloides). Neurosci Lett, 2008. 433(3): p. 241-5.

23. Hunt, G.R., R.B. Rutledge, and R.D. Gray, The right tool for the job: what strategies do wild New Caledonian crows use? Anim Cogn, 2006. 9(4): p. 30716.

24. Boeckle, M. and N.S. Clayton, A raven's memories are for the future. Science, 2017. 357(6347): p. 126-127.

25. Kabadayi, C. and M. Osvath, Ravens parallel great apes in flexible planning for tool-use and bartering. Science, 2017. 357(6347): p. 202-204.

26. Nieder, A., Evolution of cognitive and neural solutions enabling numerosity judgements: lessons from primates and corvids. Philos Trans R Soc Lond B Biol Sci, 2017. 373(1740).

27. Boeckle, M., G. Szipl, and T. Bugnyar, Who wants food? Individual characteristics in raven yells. Anim Behav, 2012. 84(5): p. 1123-1130.

28. Szipl, G., E. Ringler, and T. Bugnyar, Attacked ravens flexibly adjust signalling behaviour according to audience composition. Proc Biol Sci, 2018. 285(1880).

29. Wang, R., et al., Song control nuclei in male and female large-billed crows (Corvus macrorhynchos). Zoolog Sci, 2009. 26(11): p. 771-7.

30. Janes, S.W., The Apparent Use of Rocks by a Raven in Nest Defense. The Condor: Ornithological Applications, 1976. 78(3): p. 409-409.

31. Nunez Batalla, F., et al., [Acoustic voice analysis using the Praat program: 
comparative study with the Dr. Speech program]. Acta Otorrinolaringol Esp, 2014. 65(3): p. 170-6.

32. Aliabadi, M., R. Golmohammadi, and M. Mansoorizadeh, Objective approach for analysis of noise source characteristics and acoustic conditions in noisy computerized embroidery workrooms. Environ Monit Assess, 2014. 186(3): p. 1855-64.

33. Chang, C.L. and M.Y. Li, Predictions of Diffuse Pollution by the HSPF Model and the Back-Propagation Neural Network Model. Water Environ Res, 2017. 89(8): p. 732-738.

34. Chen, Y., et al., Prediction of benzo[a]pyrene content of smoked sausage using back-propagation artificial neural network. J Sci Food Agric, 2018. 98(8): p. 3022-3030.

35. Cao, J., et al., Big Data: A Parallel Particle Swarm Optimization-BackPropagation Neural Network Algorithm Based on MapReduce. PLoS One, 2016. 11(6): p. e0157551.

36. Ren, Z., et al., Auditory perception of geometry-invariant material properties. IEEE Trans Vis Comput Graph, 2013. 19(4): p. 557-66.

37. Zimman, L., Transgender voices: Insights on identity, embodiment, and the gender of the voice. Language and Linguistics Compass, 2018. 12(8): p. e12284.

38. Volkmann, S.S.S.a.J., The Relation of Pitch to Frequency: A Revised Scale. The American Journal of Psychology, 1940. 53: p. 329-353.

39. Mooney, T.A., et al., Loudness-dependent behavioral responses and habituation to sound by the longfin squid (Doryteuthis pealeii). J Comp Physiol A Neuroethol Sens Neural Behav Physiol, 2016. 202(7): p. 489-501.

40. Frainer, G., et al., Sound Generating Structures of the Humpback Dolphin Sousa plumbea (Cuvier, 1829) and the Directionality in Dolphin Sounds. Anat Rec (Hoboken), 2018.

41. Suzuki, H., T. Nakai, and J. Dang, Measurement of sound and vibration at the lips, nostrils, and pharynx wall in speech utterance and simulation of sound leakage from the oral cavity to the nasal cavity in nonnasal sounds. Electronics and Communications in Japan (Part III: Fundamental Electronic Science), 1992. 75(7): p. 74-86.

42. Lanzing, W.J.R., Sound production in the cichlid Tilapia mossambica Peters. Journal of Fish Biology, 1974. 6(4): p. 341-347.

43. Huber, F., CENTRAL NERVOUS CONTROL OF SOUND PRODUCTION IN CRICKETS AND SOME SPECULATIONS ON ITS EVOLUTION. Evolution, 1962. 16(4): p. 429-442. 
44. Pollack, G.S. and K. Imaizumi, Neural analysis of sound frequency in insects. BioEssays, 1999. 21(4): p. 295-303.

45. Emery, N.J. and N.S. Clayton, The mentality of crows: convergent evolution of intelligence in corvids and apes. Science, 2004. 306(5703): p. 1903-7.

46. Abbott, C.C., Intelligence of the crow. Science, 1883. 1(20): p. 576.

47. Boeckle, M., G. Szipl, and T. Bugnyar, Raven food calls indicate sender's age and sex. Front Zool, 2018. 15: p. 5.

48. Mann, S.A., et al., See no lies, hear no lies: differences in discrimination accuracy and response bias when watching or listening to police suspect interviews. Applied Cognitive Psychology, 2008. 22(8): p. 1062-1071.

49. Blecha, K.A., R.B. Boone, and M.W. Alldredge, Hunger mediates apex predator's risk avoidance response in wildland-urban interface. Journal of Animal Ecology, 2018. 87(3): p. 609-622.

50. Furrer, R.D. and M.B. Manser, The evolution of urgency-based and functionally referential alarm calls in ground-dwelling species. Am Nat, 2009. 173(3): p. 400-10.

51. Parejo, D., J.M. Aviles, and M. Exposito-Granados, Receivers matter: the meaning of alarm calls and competition for nest sites in a bird community. Oecologia, 2018. 187(3): p. 707-717.

52. Briffa, M., What determines the duration of war? Insights from assessment strategies in animal contests. PLoS One, 2014. 9(9): p. e108491.

53. Bonabeau, E., G. Theraulaz, and J.L. Deneubourg, Dominance orders in animal societies: the self-organization hypothesis revisited. Bull Math Biol, 1999. 61(4): p. 727-57.

54. Reers, H. and A. Jacot, The effect of hunger on the acoustic individuality in begging calls of a colonially breeding weaver bird. BMC Ecol, 2011. 11: p. 3.

55. Levrero, F., et al., Begging calls support offspring individual identity and recognition by zebra finch parents. C R Biol, 2009. 332(6): p. 579-89. 